\title{
Articular hypermobility simulating chronic rheumatic disease
}

\author{
R M LEWKONIA AND B M ANSELL
}

Division of Rheumatology, Medical Research Council Clinical Research Centre, Northwick Park Hospital, Harrow

SUMMARY A retrospective survey of 54 children with symptoms associated with articular hypermobility showed only occasional recognition of the syndrome before referral; some patients had been treated for inflammatory arthritis. Of the patients, $78 \%$ were girls; their mean age at onset of symptoms (11 years) was 3 years older than the mean age of the boys. Hypermobility was localised rather than generalised in most patients, and this may be an important reason for the apparent under recognition of the syndrome.

Articular hypermobility is a cardinal feature of many heritable disorders of connective tissues ${ }^{1}$ and is associated with developmental orthopaedic disorders, most notably congenital dislocation of the hip. ${ }^{2}$ Kirk et al. drew attention to a syndrome of musculoskeletal complaints associated with hypermobile joints in otherwise normal subjects. ${ }^{3}$ Of that series of 24 patients, $75 \%$ showed symptoms before the age of 16 years. Symptoms included arthralgia, joint swelling, and muscle cramps. Our retrospective survey of children with symptoms associated with hypermobility paid particular attention to features of the syndrome specific to childhood and reviewed the extent to which the syndrome had been recognised before referral.

\section{Patients and methods}

We reviewed the records of 42 girls and 12 boys aged 2 to 16 years who had been seen and examined by one of us (BMA) over a period of 6 years. They had all been referred to the Medical Research Council's Juvenile Rheumatism Unit at Taplow or the Division of Rheumatology at the Medical Research Council's Clinical Research Centre, Northwick Park. Patients with hypermobility and coincident inflammatory joint disease were not included.

Articular mobility was assessed with the procedures described by Carter and Wilkinson. ${ }^{4}$ Features sought included hyperextension of the fingers at the metacarpophalangeal joints, apposition of the thumbs to the forearms, hyperextension of the elbows or knees beyond $10^{\circ}$, and excessive mobility of the ankles (Figure). Unusual mobility of other joints found in the course of routine rheumatological examination was also noted. Height and weight were recorded on standard growth centile charts. Laboratory assays for indices of inflammatory joint disease were performed only if there were signs of possible synovitis. Laboratory tests were not necessary in many patients seen on secondary referral as normal results in the presence of what had been considered to be inflammatory joint disease were often the reason for referral.

\section{Results}

Sixty of our patients had Caucasian parents, one boy was Chinese, one boy had an Iranian father, and one girl had an Asian mother and a Caucasian father who had craniocleidodysostosis. Features of heritable disorders of connective tissue were sought, but only two patients had definite features of such diseases. The Ehlers-Danlos syndrome type III was diagnosed in a girl who developed dislocated shoulders at 3 months, dislocated hips at 4 years and had a striking family history of articular hypermobility. A 15 year old girl was considered to have the marfanoid hypermobility syndrome ${ }^{5}$ because she had arachnodactyly and other features of Marfan's phenotype; her height was at the 75th centile and her weight at the 3 rd centile.

Two girls were training in gymnastics, one spending as much as $\mathbf{3 0}$ hours a week in training for competitive gymnastics. A further two girls were attending ballet school. Several of the older children dated the onset of their symptoms to minor sports injuries. Referrals came from a variety of medical 

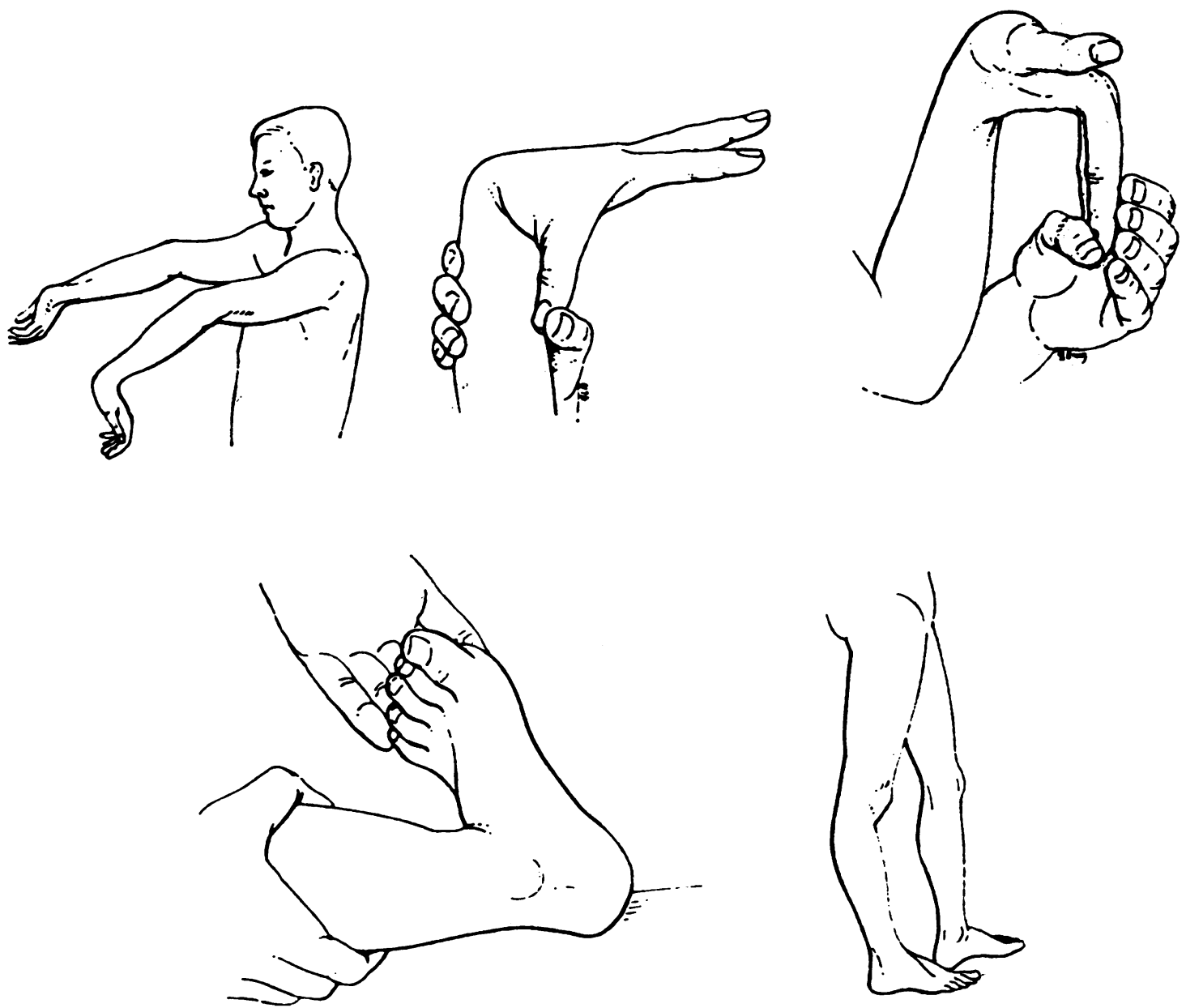

Figure Procedures for showing articular hypermobility. ${ }^{4}$ (From Wynne-Davies, ${ }^{2}$ by kind permission of the author and the editor of The Journal of Bone and Joint Surgery.)

sources including 12 from paediatrics departments, 7 from rheumatology and rehabilitation departments, 5 from orthopaedic departments, and 30 from general practice. Hypermobility had been suggested as a diagnosis in only six of these cases. Three patients had already undergone arthroscopy of the knee, and another had undergone patellar shaving for presumed chondromalacia patellae. Many patients had received prescriptions for aspirin and non-steroidal anti-inflammatory drugs, and, of the patients previously seen by rheumatologists, two had been treated with systemic steroids and one with penicillamine after simpler antirheumatic agents had proved ineffective.
The mean age at onset of symptoms in boys was 7.71 years, which was significantly younger than the mean age at onset of symptoms in girls $(10.98)$ $(P=0.047)$. Table 1 shows the distribution of heights and weights with the mean height and weight centiles for boys lower than those for girls. Regression analysis of height and weight centiles for individual patients showed the lines for boys and girls to be parallel, but the regression line for girls was significantly higher than that for boys $(P=0.043)$. Ranking of the distribution of affected joints showed the knees to be most commonly affected by both symptoms and signs (Table 2). The mean age at onset of symptoms of back pain was 
Table 1 Age and stature of patients with articular hypermobility

\begin{tabular}{|c|c|c|}
\hline & Boys & Girls \\
\hline \multicolumn{3}{|l|}{ Age at onset of symptoms: } \\
\hline $1-5$ years & 4 & 4 \\
\hline 6-10 years & 3 & 12 \\
\hline 11-16 years & 5 & 26 \\
\hline Total & 12 & 42 \\
\hline Mean age (SD) at onset (years) & $7 \cdot 71 \quad(4 \cdot 83)$ & $10.98 \quad(3.41)$ \\
\hline $\begin{array}{l}\text { Mean centile height (SD) when } \\
\text { examined }\end{array}$ & $35.45(21.99)$ & $58 \cdot 35(25 \cdot 22)^{*}$ \\
\hline $\begin{array}{l}\text { Mean centile weight (SD) when } \\
\text { examined }\end{array}$ & $38 \cdot 91(22 \cdot 78)$ & $50.8(27 \cdot 78)^{*}$ \\
\hline
\end{tabular}

*Excludes patient with Marfan's syndrome.

Table 2 Articular distribution of symptoms and abnormal joint mobility in boys and girls with articular hypermobility

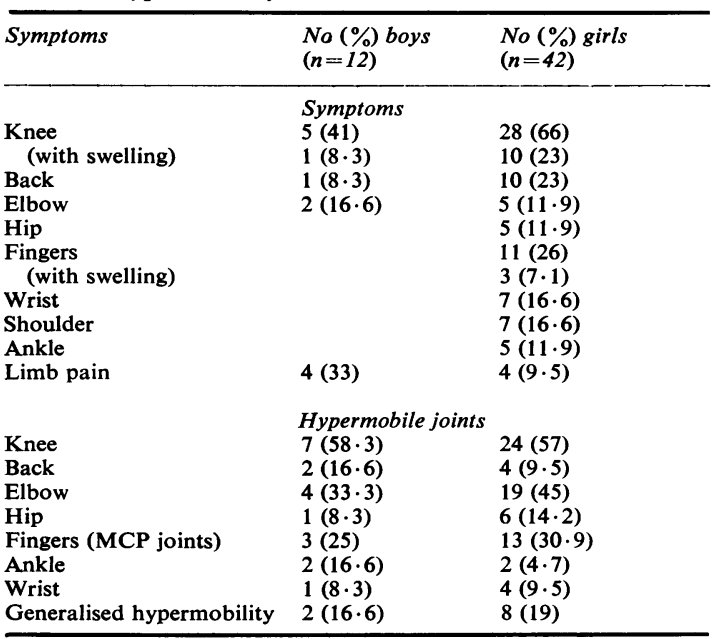

13.2 (SD 1.59) years, and all but one of the 11 patients with back pain were girls. The boy with back pain and one of the girls were later found on $x$ ray examination to have Scheuermann's osteochondritis.

\section{Discussion}

The presence of articular hypermobility as the basis of presenting complaints had been recognised occasionally in our series of patients referred by paediatricians and other specialists as well as by general practitioners. Patients in whom hypermobility had been recognised would probably not be referred, and our series is therefore likely to show an ascertainment bias. Nevertheless, the hypermobility syndrome seems to be under recognised, ${ }^{6}$ and this may lead to unnecessary investigation or treatment for inflammatory joint disease. ${ }^{7}$ The apparent tendency to mistake hypermobility for inflammatory joint disease in these patients may reflect lack of awareness of the syndrome but may also be a consequence of the difficulties of assessing abnormal joint mobility in childhood. Criteria for diagnosis of generalised hypermobility were developed by Carter and Wilkinson, who reported abnormality in $7 \%$ of 285 normal school children aged 6 to 11 years. ${ }^{4}$ Articular mobility declines progressively throughout and after childhood, ${ }^{289}$ and the criteria have been found to be both sensitive ${ }^{10}$ and insensitive ${ }^{11}$ at different ages in population surveys. In the Scottish survey of Wynne-Davies $65 \%$ of subjects had hypermobile wrists at 2 years old, falling to only $5 \%$ at 7 , and $30 \%$ had hypermobile knees at 18 months, falling to $5 \%$ at $3 \frac{1}{2}$ years. ${ }^{12}$ In addition to variation with age and anatomical site, ${ }^{13}$ joint mobility also tends to be greater in females than males in all age groups. ${ }^{8}$ Thus the well known criteria of Carter and Wilkinson ${ }^{4}$ for generalised articular hypermobility must be applied in the context of the age and sex of the patient and may not be fulfilled in many who, nevertheless, have localised hypermobility.

It follows from previous population surveys that, hypermobility being common, inflammatory arthritis may precede or coexist with both symptomatic and non-symptomatic hypermobility, and this is a potential source of diagnostic confusion that merits special consideration. Clinical features that would suggest 'benign hypermobility' rather than inflammatory joint disease include the absence of systemic illness or rash, absent or limited joint swelling, large and medium sized joints predominantly affected rather than small joints, and absence of synovial or soft tissue thickening despite chronicity of symptoms. Muscle cramps and limb pain that are not localised to joints and occur after strenuous exertion are unusual in inflammatory joint diseases but common in this syndrome. A family history of 'loose jointedness' in childhood is also useful as circumstantial evidence in differential diagnosis. Negative or normal laboratory results will sustain the diagnosis of hypermobility, which is essentially one of exclusion. If there is persistent synovial effusion, even with normal laboratory results for inflammatory and infective disease, it is diagnostically helpful to show the absence of pleocytosis in the joint fluid to be confident that a hypermobile child does not also have arthritis.

Bruising or wide scars should prompt examination of skin elasticity and consideration of the EhlersDanlos syndrome. Only two of our patients had definite features of heritable syndromes of abnormal 
connective tissue. In this regard our findings do not concur with those of a recent report ${ }^{14}$ that described 9 patients from five families with the Ehlers-Danlos syndromes types I, II, and III who were referred to a paediatric rheumatology clinic over a period of 6 months. The relation of the commonly occurring hypermobility of childhood to the classical EhlersDanlos syndrome, especially the mild type III variety, is likely to remain uncertain until a specific biochemical abnormality is shown in the EhlersDanlos syndrome types I, II, and III.

The prevalence of symptomatic hypermobility cannot be assessed from this retrospective survey because of the selected nature of the referred patients, but it is clear that only a minority of children with hypermobility develop symptoms. The mechanism of symptoms in those who do manifest the hypermobility syndrome are unknown, and the syndrome does not appear to correlate with the extent of hypermobility. Indeed, children with gross hypermobility associated with various types of the Ehlers-Danlos syndrome may have symptoms due to instability of weight bearing joints but they rarely develop joint effusions or the musculoskeletal complaints of the hypermobility syndrome (R $M$ Lewkonia and $F$ M Pope, unpublished observations). The onset of symptoms in some of our older patients followed excessive or unaccustomed activities in physical education or sports. The preponderance of girls in this series and their tendency to be slightly taller than expected for their age are also of interest. These observations suggest that symptoms arise when ligaments and connective tissues are subjected to mechanical stress inappropriate to the stage of maturity that the individual has reached; this progressively constrains joint mobility. It remains difficult to explain the pathogenesis of joint swelling, the persistence of symptoms, or the non-articular limb pains noted in our patients or in the original series of Kirk et al. ${ }^{3}$

Psychological problems appeared to be important in perpetuating symptoms in some of our patients, but it was difficult to establish that such factors were not more common than would be expected in a suitably matched control population. Several of our patients had family histories of either juvenile chronic or adult arthritis, and, not unexpectedly, the development of joint or limb pains in another member of the family generated considerable anxiety. Back pain was predominantly a symptom of older girls, a group also especially susceptible to psychogenic limb pain. ${ }^{15}$ Failure to recognise the hypermobility syndrome was an important factor in perpetuating symptoms, which, in a few patients, had led to counterproductive invasive orthopaedic investigation or the use of potent antirheumatic drugs. The most important factor in management was often careful explanation of the nature of symptoms associated with hypermobility together with reassurance that the symptoms were not a prelude to juvenile chronic arthritis.

In most of the patients available to us for follow up symptoms had resolved within a year or two of the diagnosis being established and explained. Persistence of symptoms necessitates reinvestigation with consideration of both psychosocial circumstances and the possibility of slowly evolving disease processes. The long term prognosis of this syndrome requires elucidation, and this can only be achieved with very lengthy longitudinal studies. The important question of whether the hypermobility syndrome predisposes a subject to premature osteoarthritis ${ }^{3}$ has to be considered in the context that articular hypermobility is not a homogeneous entity. The long term consequences of hypermobility were not addressed in the present study, but our observations in other patients suggested that individuals with a childhood history of articular hypermobility may present in middle age with osteoarthritis developing at distinctive sites, namely the carpometacarpal of the thumb, patellofemoral joint, and joints of the midcervical spine.

We thank Stephen Duffy, Division of Computing and Statistics, Clinical Research Centre, for help in statistical interpretation. RML was in receipt of an Associateship of the Canadian Arthritis Society.

\section{References}

1 Hollister DW, Byers PH, Holbrook KA. Genetic disorders of collagen metabolism. Adv Hum Genet 1982;12: $1-87$.

2 Wynne-Davies R. Acetabular dysplasia and familial joint laxity: two etiological factors in congenital dislocation of the hip. J Bone Joint Surg $[\mathrm{Br}] 1970 ; 52 B$ : 704-16.

3 Kirk JA, Ansell BM, Bywaters EGL. The hypermobility syndrome. Musculoskeletal complaints associated with generalized joint hypermobility. Ann Rheum Dis 1967; 26:419-25.

4 Carter CO, Wilkinson J. Persistent joint laxity and congenital dislocation of the hip. J Bone Joint Surg $[\mathrm{Br}]$ $1964 ; 46 B: 40-5$

5 Walker BA, Beighton PH, Murdoch JL. The marfanoid hypermobility syndrome. Ann Intern Med 1969;71: 349-52.

- Biro F, Gewanter HL, Baum J. The benign hypermobility syndrome in pediatrics. (Abstract) Arthritis Rheum 1982;25(Supplement, April):S144.

7 Bird HA, Wright V. Joint hypermobility mimicking pauci-articular juvenile polyarthritis. $\mathrm{Br}$ Med J 1978;ii: 402-3.

8 Beighton P, Solomon L, Soskolone CL. Articular mobility in an African population. Ann Rheum Dis 1973; $32: 413-8$. 
9 Moran HM, Hall MA, Barr A, Ansell BM. Spinal mobility in the adolescent. Rheumatol Rehab 1976;18: 181-5.

10 Bird HA, Brodie DA, Wright V. Quantification of joint laxity. Rheumatol Rehab 1979;18:161-6.

11 Silverman S, Constine L, Harvey W, Grahame R. Survey of joint mobility and in vivo skin elasticity in London school children. Ann Rheum Dis 1975;34:177-80.

12 Wynne-Davies R. Familial joint laxity. Proceedings of the Royal Society of Medicine $1971 ; 64: 689-90$.

13 Wood PHN. Is hypermobility a discrete entity? Proceedings of the Royal Society of Medicine 1971;64: 690-2.
14 Osborn TG, Lichtenstein JR, Moore TL, Weiss T, Zuckner J. Ehlers-Danlos syndrome presenting as rheumatic manifestations in the child. $J$ Rheumatol 1981 ; 8:79-85.

15 Apley J. Limb pain with no organic disease. Clin Rheum Dis 1976;2:487-91.

Correspondence to Dr B M Ansell, Division of Rheumatology, Clinical Research Centre, Northwick Park Hospital, Watford Road, Harrow, Middlesex HAl 3 UJ.

Received 15 August 1983

\section{British Paediatric Association}

Annual meetings

$\begin{array}{lrl}1984 & 10-14 \text { April } & \text { York University } \\ 1985 & 16-20 \text { April } & \text { York University } \\ 1986 & 15-19 \text { April } & \text { York University } \\ 1987 & 7-11 \text { April } & \text { York University }\end{array}$

